festations of reflex nervous irritation, though they may exist in a severe form without producing any such result.

Of the nervous and complicated morbid conditions produced from dental diseases, the following may be mentioned as occurring less frequently than those above mentioned : Chronic trismus, epilepsy, tetanus, partial paralysis, amaurosis, deafness, opthalmia, and perverted nutrition.

It is not deemed necessary to occupy your time in the narration of cases illustrating the foregoing observations, but I will refer you to the works of Salter, Bond, Garrison, and our periodical literature, where they may be found and read at your leisure.

From the foregoing observations, it is surely very important that the physician should never disregard the dental orgaus as agencies in the production or continuance of diseases; and it should also deeply impress the dental practitioner with the importance of a clear and comprehensive knowledge of the entire organism, both in health and in a diseased condition. He should ever bear in mind that his operations are not upon isolated organs merely, but upon organs bearing very intimate and close sympathetic relations to the entire economy.

PISTOL-SHOT WOUND OF THE ABDOMEN; LAPAROTOMY AND SUTURE OF THE WOUNDED INTESTINES.

Read before the Medical Society of Virginia, October 22, 1887 , BY A. S. PRIDDY, M.D., of KEYSVILLE, VA.

Ras Wimbish, a negro, aged 60 years, was twice shot by his employer, with whom he was engaged in an altercation, on September 4, I887, about 8 P.M., with a Smith \& Wesson revolver, 32 calibre, at a distance of six or eight feet. The shooting occurred on a farm in Mecklenburg county, about four miles from the town of Chase City. The wounded man, at the last discharge of the pistol, exclaimed that he was shot, ran to the stables, mounted a horse, and rode into town where his family lived, "to get a doctor to cut the balls out." Dr. C. W. Walker was called in, and found him falling into a state of profound shock, from which it was several hours before he began to rally. He was, after recovering from the shock, very restless, with an anxious countenance, much thirst, pulse quick and weak, and hiccoughed slightly, though vomited none. Dr. Walker administered $1 / 3$ grain of morphia, and proceeded to examine him.

He found that the first shot had entered the fleshy portion of the thigh, and passing through was lodged under the skin on posterior aspect, from which it was easily extracted. The second shot had taken effect about one-half inch below the anterior superior spine of the ilium, toward the abdomen, the point of entry being small, presenting a jagged appearance, and was sealed with a clot of blood. Abdominal palpation revealed nothing wrong, except considerable pain on pressure.
He complained of an excruciating pain in abdomen. The track of the ball was not fully explored with a metallic probe, as it was quite certain, from its direction, that it had entered the abdominal cavity. The man was put to bed and the usual "ostrich plan" of treatment pursued; that is, warm applications to the bowels, and sufficient opium to control pain, to await developments in the case.

On the next morning the patient was found to have passed a fairly comfortable night, under opium. He had voided a large quantity of clear urine, but his bowels had not moved since the shooting. The abdominal pain and tenderness had increased. The abdomen had become very tympanitic and emphysematous, which symptoms were attributed to a gaseous distension of the abdominal walls, or of the intestines. This condition was of such importarce as to make death seem imminent from dyspnœa.

Thus things continued: dyspnœa, hiccough, occasional retching and vomiting, slightly elevated $t \in m$ perature, and an obstinate constipation, till the third day after the shooting, when a cathartic was given wnich in due time produced several evacuations, containing considerable blood. This made intes. tinal perforation certain beyond cavil. After this the tympanitic condition of the abdomen disappeared considerably for nearly twenty-four hours, after which the before-mentioned condition again became just as alarming, and so continued.

On September 8th, the fourth day after the shooting, Dr. F. J. Gregory, of this place, and I were telegraphed for. We arrived about 9 o'clock that night, and found the man in the condition before mentioned; and according to Dr. Walker he was growing weaker. There were also slight peritonitic symptoms. We advised an exploratory incision, with several objects in view: first, to relieve the gaseous distension, which was fast causing his death from dyspncea; to cleanse the cavity of all extravasations and to repair damage to the wounded viscera; and give free drainage, which might lessen his chances of death from suppurative peritonitis, septicæmia, etc., or at least make his time of living more comfortable, and his death one not attended with so much suffering. These views were concurred in by Dr. Walker, though we thought that the "golden opportunity" had been lost by waiting so long. Yet, at earnest solicitation of the wounded man to give him every known chance for his life, however slight that chance might be, we decided on a laparotomy the next morning, provided the man were living.

The operation was performed on September $g^{\text {th, }}$ the patient being still alive. I made preparations for the operation, which were about as complete as could be made in a negro cabin, but not so elegant as some of our well equipped city hospitals afford. The instruments were placed in a 5 per cent. solution of carbolic acid, and the sponges thoroughly washed out in that solution. The hands and nails of all the participants in the operation were cleansed with soap and water, and then dipped in carbolized oil. The toilet of the abdomen was made by scouring the walls, shaving the pubes, and washing the whole over with a 5 per cent. solution of carbolic 
acid, especial attention being paid to the navel and point of entry of the ball.

The sponges were next placed in bichloride solution in water $1-1500$. Plenty of towels were also provided in same warm solution.

At to o'clock, in the presence of and assisted by Drs. Gregory, Walker, H. H. Jones, W. B. Jones, and Mr. Ogburn, after the hypodermic administra. tion of $1 / 3 \mathrm{gr}$. of morphia and I- $100 \mathrm{gr}$. atropia, chloroform was administered and the operation begun. An incision was made in the median line from the umbilicus to the symphisis pubis, down to the peritoneum, which was incised after the capillary bleed. ing had been stopped by pressure with sponges wrung out of hot bichloride solution. On opening the peritoneum a considerable quantity of bloody serum, pus, etc., escaped from the cavity, all of which had a distinct and disagreeable foecal odor; yet there was only a trace of focal matter in the cavity. I then introduced my hand into the cavity and slowly drew out the intestines, examining each coil carefully till I came to the jejunum, on whose mesentery I noticed a contusion about the size of a silver dime, which was evidently the point at which the force of the ball had spent itself. When the descending colon was reached it was found to be lacerated longitudinally for more than six inches, extending into the sigmoid flexure. I passed my whole hand through the wound in this intestine, into the rectum. The meso.colon was also found perforated. The intestines were wrapped in towels wrung out of the hot bichloride solution, during the operation, and the normal temperature preserved so far as possible.

The lacerated gut was united by a continuous suture of catgut, which was improvised by soaking a fiddle string in water the night before, and then soak. ing a while in carbolized oil. The abdominal cavity was washed out with the bichloride in water $I-1500$, and then thoroughly sponged out.

The wounds, both in the gut and mesentery, were packed with iodoform. I did not think it necessary to excise the wounded mesentery. The intestines, after having been thoroughly cleansed, were returned to the cavity. A Nèlaton's soft rubber catheter, No. r2, was "nicked" in several places, and introduced into cavity as a drainage tube. The abdominal incision was closed with seven sutures of white silk, which included the peritoneum. Adhesive strips were then applied to the abdomen. The wound was dressed with iodoform, and a large cotton pad covered the whole abdomen.

The operation lasted about fifty five minutes, which was a rather shorter time than I intended to take, but the man came near dying under the operation, and was only stimulated by several hypodermic injections of arom. spts. ammonia and brandy, and, with a little speed in the operation on my part, came out very well. He was immediately put to bed, and hot rocks applied to his extremities and epigastrium. Whiskey or brandy were given freely; and in due time reaction came on freely. He was ordered milk and raw eggs for food, and this food alone, with stimulants of whiskey or brandy when needed. Morphia in $1 / 4 \mathrm{gr}$. doses was ordered every three or four hours, to control pain and lock up his bowels.

At 3 o'clock P.M. that day his temperature was $100^{\circ}$, pulse I IO, respiration normal and easy; slight hiccough; no tympanites; lays with legs extended, suffered little pain.

Sept. ro, 8 A.M. - Seen by Dr. Walker. Temperature $100^{\circ}$, pulse 135 . Tongue coated, anxious countenance, no peritonitic symptoms. Spent a moderately comfortable night under morphia. Think he may live through to-day. Dr. Walker was summoned before the court to testify in the case and did not see him again until 6 P.M., when he found him almost completely exhausted, pulse about 180 , no volume, and scarcely perceptible. Skin bathed in a cold, clammy sweat, extremities very cold. Did not think it possible for him to live through the night. Ordered milk and whiskey every few minutes through the night, and hot rocks to his feet.

Sept. i I, 8 A.M.-Seen by Dr. W., who found a marked improvement in his condition; had spent a very comfortable night when not taking the nourishment. Temperature normal, pulse 135. respiration free and easy, extremities warm. No peritonitis. Could turn over in bed. Took nourishment of milk and whiskey regularly and with apparent relish. Some little colicky pains in abdomen relieved by morphia. He continued to do well throughout the day, and took a supper of rice, contrary to our directions.

Sept. 12, 8 A.M.-Rested well last night under morphia. Temperature normal, pulse 120 , with good volume. Tongue clean, no peritonitis, suffering no pain; the abdominal wound suppurating quite freely, drainage-tube doing good work.

6 P.M.-Dr. Walker found him doing well. No change in condition except that he had a large solid evacuation of the bowels, which caused very little pain or tenesmus; contained no blood nor mucus.

Sept. 13, 8 A.M. - Seen by me for the first time since the operation. Found him comparatively comfortable, temperature normal, pulse roo, full and strong. Could turn over in bed or lie on either side. Kept flies off himself. I removed the dressings from the abdomen and found that the abdominal wound had united entirely down to fourth suture. Washed the abdomen off with carbolized water and applied clean cotton, after dressing the wound with iodoform. He did well the whole day.

Sept. I 4, 8 A.M.-Seen by Dr. Walker. Rested well the night before with $1 / 4$ gr. morphia. Had taken sufficient food of milk and eggs. Temperature normal, pulse roo, good volume; had a solid evacuation, which griped him considerably.

Sept. I 5, 8 A.M.- Did not take any morphia last night, and spent quite a restless night. Some little tenderness over bowels, but doing well in other respects. Left a dose of morphia for him to take at night.

Sept. I6, 8 A.M.-Seen by Dr. Walker. Rested well last night. Comfortable, pulse Ioo, temperature normal, tongue glazed and red, extremities a little cold. Noticed a continuous discharge, of a muco purulent nature, from the anus. 
Sept. I 7, 8 A.M.-Seen by Dr. Walker and me. Found him beginning to show some signs of exhaustion. Pulse r ro and feeble, temperature subnormal, extremities rather cold; all of which symptoms were due to the poor attention to his food and other nursing, which his ignorant and indifferent family gave. Dr. W. B. Jones consented to nurse and feed him properly, which he did, and marked improvement at once followed.

Sept. 19, 8 A.M.-Doing well. Wound entirely united. Removed drainage-tube and took out all of the sutures and applied adhesive strips. Takes food of milk, eggs, peptonized beef, soups, etc.

Sept. 20.-Doing well, and from this time the improvement was steady and uneventful, he being able to take solid food. Sleeps well and has sat up on one or two occasions. The point of entry of bullet commenced suppurating about October I, and was very superficial, being due to some little foreign substance being carried in, and was relieved by syringing the hole out with a 3 per cent. solution of carbolic acid in water.

I saw him on October I 2 , which was a few days prior to the writing of this report, and found him, to all outward appearances, entirely well of the wound and the operation, but feeble, and suffering at the time from an attack of diarrhœa which, I think, was in no way dependent on the wound, but rather to be attributed to some improper article of food given him, and to bad nursing in general. His age, 6o years, the poor attention paid him by his family, who are entirely indifferent as to his ultimate recovery, and the confinement in the house and bed, tend to make his recovery of former strength and activity necessarily slow. But as I have said before, at this time, nearly six weeks after the shooting, he is practically weli of the injury.

Remarks.-Although the profession is much interested in this branch of surgery, yet the literature on it is of necessity very meagre, for it was only in I $88 \mathrm{I}$, that Dr. R. A. Kinloch, of Charleston, S. C., first performed laparotomy for gunshot wounds of the abdomen; and only in 1883 , that Kocher, of Berne, and 1884 that Dr. W. T. Bull, of New York, performed the first two successful cases on record. I have not mentioned the case reported and recorded in the "Medical and Surgical History of the War of the Rebellion," which Dr. Kinloch performed in I 863 on a confederate soldier for a gunshot wound sustained seven months previous to the operation, and which was entirely successful, the man living several years after the war; but I think this operation was more for disease than for traumatism, and should so be regarded.

Of all the cases of laparotomy reported-and I think there are only fifty-seven-I think my case presents some points of interest which are not seen in the others. There may have been more viscera injured in some other reporled cases, and I think Dr. Keen, of Philadelphia, reported last April a case in Vineland, N. J., in which the stomach, colon, ileum and kidney were wounded, from which the pa tient died on the fifteenth day. There are also sev eral other cases in which the stomach was perfor- ated, and another in which the liver was wounded. But my case presented a larger wound in the intestine, and was operated on after the fifth day of the injury, and the patient was just being attacked with peritonitis; and of which tended to make the man's chances of recovery infinitesimally small.

One remarkable feature in the case is, I think, the very small quantity of foecal matter found in the cavity; which can be explained by saying that the man ate an early dinner, and had eaten no supper. so nearly eight hours had elapsed since the last food was taken, and consequently the contents of the bowels had sought the lowest portion of the tract, and had passed to a part below the site of injury. The bowels were evacuated in the early part of that day.

My case and the case of Dr. Kollock, of Cheraw, S. C., are the only two cases that have been opererated on in negro cabins, where the surgeon labors under a great many disadvantages. The negroes are, as we all know, very ignorant and careless, and good after-treatment is from them an impossibility. And in the country it is very inconvenient and sometimes impossible for the surgeon to see his patients so often as necessary.

I have reported this case merely to show under what unfavorable circumstances this operation may be undertaken, and to what a satisfactory terminait may be brought; for were the man to die now the operation has certainly saved him six or seven weeks of life, when, on the contrary, it would have been impossible for him to have lived but a few hours without the operation, for focal extravasation would have been almost sure to have followed, and as I have said before, peritonitis had begun to set in. Even if the wounds be of such gravity as to make recovery impossible, as Dr. Kinloch well says, "next to the cure of disease we look to the satisfaction coming of a painless death" by this operation. The patient will certainly die an almost painless death, which will well repay any one for the trouble and risk in undertaking the operation.

I hope that this case, with other reported cases, may encourage any and every physician, whether living in the city or country, to lay aside the old passive line of treatment, and offer to a man with any abdominal traumatism zhatever, the chances for his life offered in this day of antiseptic surgery; and not until he has done this will he be free from all responsibility.

\section{A CASE OF EXTIRPATION OF THE KIDNEY, WITH REMARKS. ${ }^{1}$}

BY L. H. DUNNING, M.D., OF SOUTH BEND, $1 \mathrm{ND}$.

The patient, Mrs. H., aged 46 , underwent the operation of nephrorraphy Oct. $3 \circ, 1884$. The history of this case was published in Feb., 1885,2 The kidney was fixed at the time of the operation by eight sutures, three of which passed through and included the renal

1 Read by Title before the Indiana State Medical Scciety.

2 Thr Journal, Feb. $2 x, 1885$. 